\title{
Smarter Way to Access Multiple Mobile Cloud Applications without Interoperability Issues
}

\author{
P Thanapal $^{\mathrm{a}}$, K Marimuthu ${ }^{\text {,** }}$, S Rajkumar ${ }^{\mathrm{b}}$, R Niranchana ${ }^{\mathrm{b}}$ \\ ${ }^{a}$ School of Information Technology and Engineering, VIT University, Vellore-632014, India \\ ${ }^{b}$ School of Computer Science and Engineering, VIT University, Vellore-632014, India
}

Received: 06 March 2017; Accepted: 18 June 2017; Published: 08 September 2017

\begin{abstract}
As we are in the world of emerging technologies people have more awareness about it especially Smartphone's. Usage of Smartphone's has been increased tremendously. Different operating systems (OS) are used in smart phones such as MAC OS, Android, and Windows OS etc. Most of the applications are run on their appropriate platform. Cloud is the simplest way to run certain application over cross platforms. Many cloud vendors are there who provides several kinds of applications and software which can be accessed by the users. In that Application Programming Interfaces (APIs) helps us to create a cloud application over the market. However there will be chances to occur Interoperability issues while users were working on a cross platforms. In order to overcome this issue a framework has been proposed named Mobile Cloud Middleware (MCM). MCM will recover the interoperability issues over multiple cloud services on mobile devices. Here in this paper we proposed effective result to solve the Interoperability issue by integrating Mobile Enterprise with MCM.
\end{abstract}

Index Terms: Mobile Cloud Application, Application Programming Interface, Mobile Cloud computing, Mobile Cloud Middleware, Mobile Enterprise.

(C) 2017 Published by MECS Publisher. Selection and/or peer review under responsibility of the Research Association of Modern Education and Computer Science

\section{Introduction}

In day to day life most of the people were using Smartphone's because of its advanced features. One of the best features in Smartphone is cloud applications. Cloud applications are nothing but applications which are run over cloud environment called Cloud Computing [1]. Cloud and Mobile Cloud Computing has become more popular. Cloud Computing is a concept of connecting a large number of system or servers over real time * Corresponding author.

E-mail address: 
network such as internet for communication. Cloud provides services such as running cloud applications provided by cloud vendors, data storage, managing, maintenance etc.

In cloud environment user needs to access multiple tasks among mobile cloud data, so that users may face a kind of problem known as interoperability issues. In order to avoid such issues Mobile Cloud Middleware is used. Mobile Cloud Middleware is the key to solve the issues that are facing over the multiple cloud approaches. In this research paper we also discussed about offloading data, which occurs large amount of computational processing and how it will be handled easily with the help of MCM. And also MCM has an asynchronous invocation over the mobile cloud service which leads to improve the quality of service for mobiles to managing its responses.

\section{Cloud Computing vs Mobile Cloud Computing(MCC)}

Cloud Computing assures virtual infinite resources. It also includes three levels of services such as Software as a Service (SaaS), Infrastructure as a Service (IaaS) and Platform as a Service (PaaS). Cloud Computing is a paradigm in which we can store a data permanently in the server [2]. Then the stored data can be cached temporarily by client as they need. It is an internet based approach where we can store data, share resources, etc. it may also allow user to modify their data which stored in cloud [3]. Mobile Cloud Computing is differs from Cloud Computing. MCC is simplest form refers to an infrastructure. MCC allows Mobile Cloud Application to compute power which leads to data storage, energy storage. It also makes the mobile range much broader for mobile subscribers. Figure 1. shows the processing power and data storage in MCC.

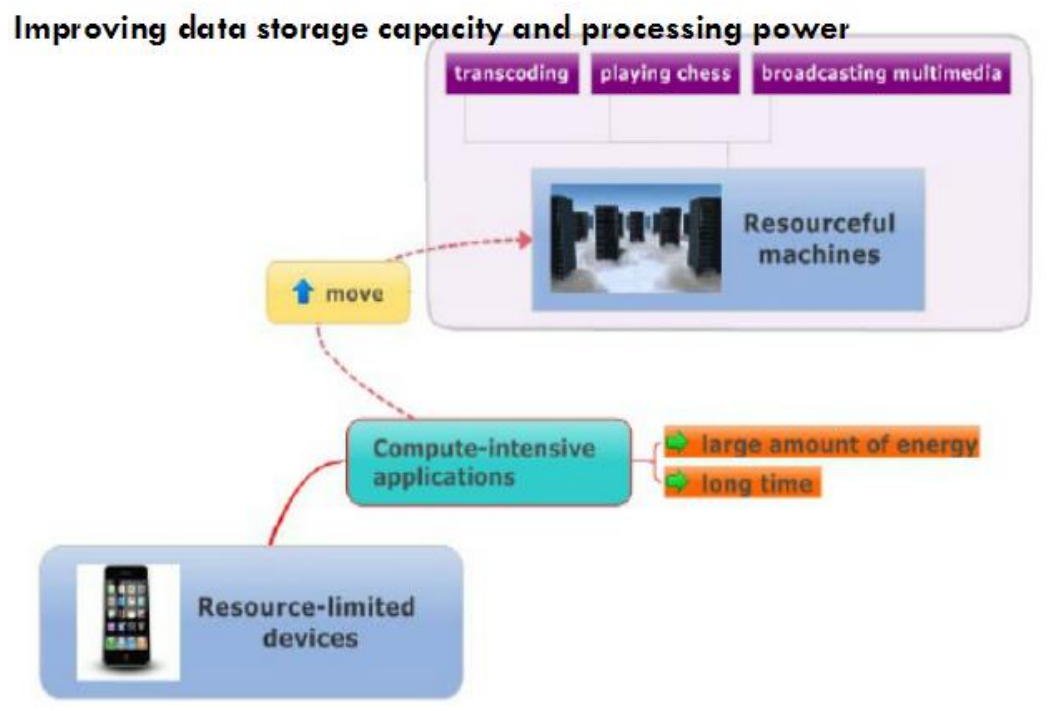

Fig.1. Processing power and data storage in MCC

\section{Mobile Cloud Middleware}

Mobile cloud allows the users to process intensive task which are done by Smartphone's and also to store it in a domain. Smartphone's focused on cloud in order to enrich the features of its own. It makes use of cloud to mash up applications. Mobile Cloud Middleware become popular because most vendors provides synchronisation services and also cloud allows to store centralized data resources like gmail, images, contacts details in it. Mobile Cloud Middleware is based on the data synchronization approach. The main function of this approach is to process data intensive tasks [4]. 
Mobile Cloud Middleware acts as an intermediary between both cloud and mobile device. When a device tries to connect with cloud first it connects to TP-Handler [5] which situated in middleware. Then the request sends to MCM Manager adapter which leads to transaction process. Fig 2. shows mobile cloud architecture.

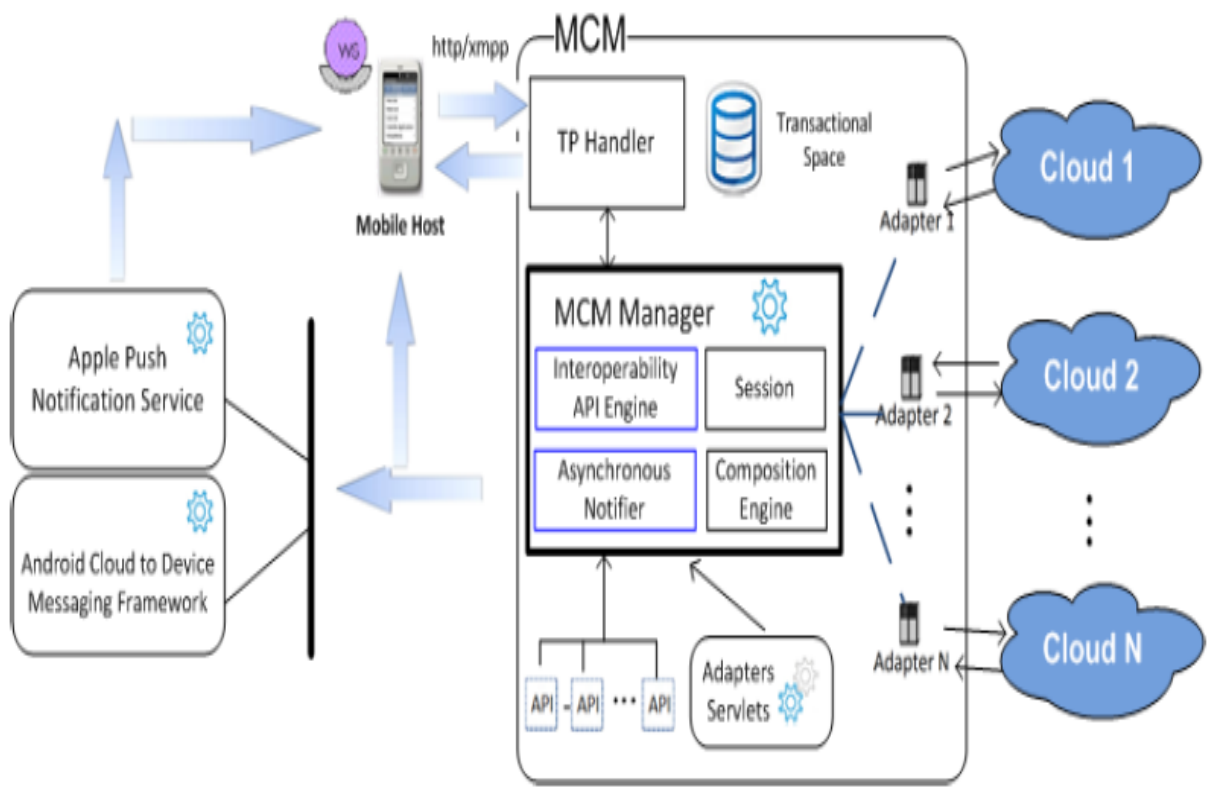

Fig.2. Mobile cloud architecture

Mobile Cloud middleware consists of push notification and mobile host technologies for processing remote executions. Mobile host technology [6] provides asynchronous mechanisms. And also this mechanism has a certain limitation and constraints for processing a certain platform. The following figure will represents how the mobile host mechanism being processed.

Mobile cloud Middleware leads to asynchronous mechanisms in it. It also helps the mobile application to manage the cloud services. An example is Android, IOS concepts which are being used by cloud services. Let see with the help of Bakabs how the cloud services are managed. Goole analytics provides the details about how many times a single profile is loaded. By taking the input it starts linear programming task [7]. Then it accesses the GDATA by making use of API. Once the result is published then it sent it back to mobile device.

Mobile Cloud Middleware are consists of 4 major categories. They are Reflective, Tuple Space, Context Aware and Event-Based middleware [8,9]. Each category may supports requirements imposed by mobility. Let see the differences between the categories among requirement based. Fig 3. shows mobile host in MCM. 


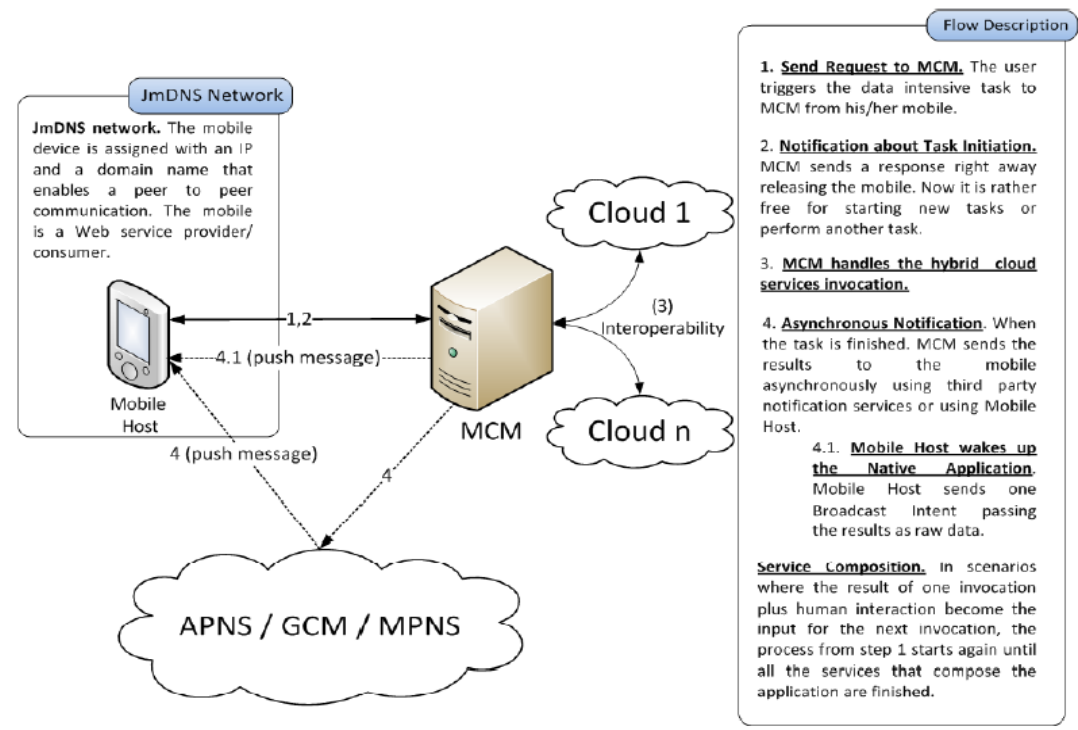

Fig.3. Mobile host in MCM

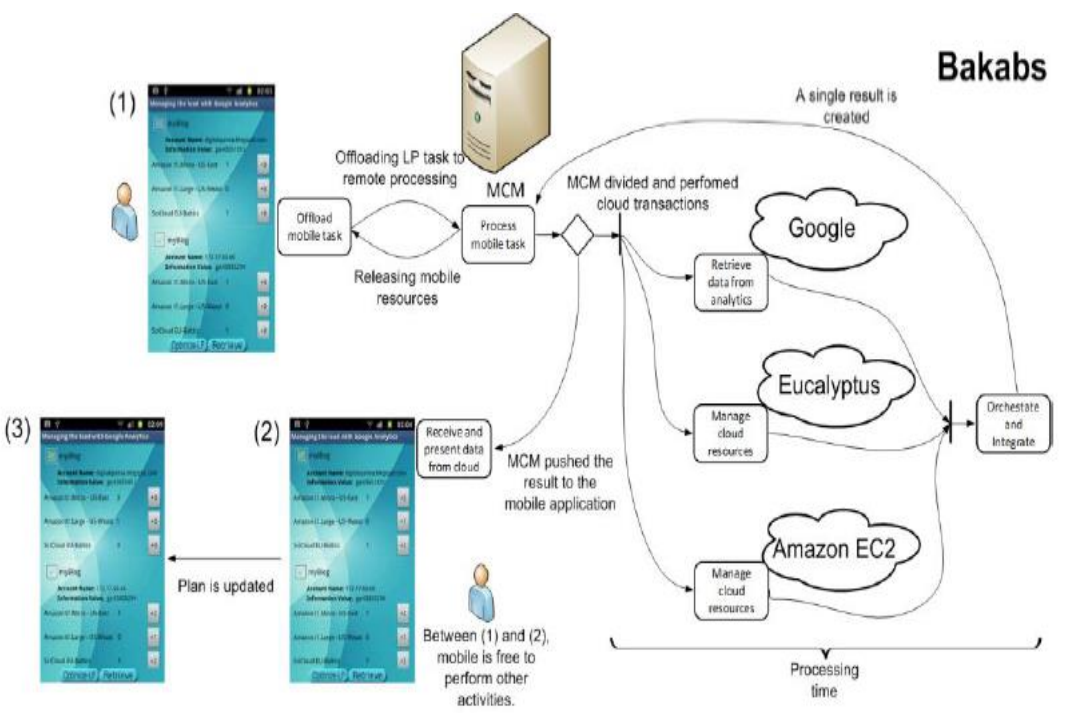

Fig.4. Linear processing in cloud

Table 1. Categories and requirements

\begin{tabular}{|c|c|c|c|c|c|}
\hline & Awareness & Adaptation & Synchronous & Asynchronous & Re Configuration \\
\hline Reflective & Yes & Yes & Yes & - & Yes \\
\hline Tuple Space & - & - & - & Yes & - \\
\hline Context Aware & Yes & Yes & Yes & - & - \\
\hline Event Based & - & - & - & Yes & - \\
\hline
\end{tabular}


Table 1. tells that Awareness, Adaptation and Synchronous are the requirements needed for Reflective and Context Aware middleware technologies. The Tuple Space and Event Based Technologies needs the only requirement is Asynchronous. Fig 5. shows the linear processing in cloud.

\subsection{Reflective Technology}

Reflective Middleware is normally used in programming language in order to design more extensible languages. Main principle of Reflection technique is to access the program and make changes of its own behaviour. It may also used in Operating Systems.

Reflection Middleware have 2 types of levels.

\section{(i) Meta level:}

It can be accessed through reification i.e. exposing internal representation which is in the state of hidden. It leads to access the base level application.

(ii) Base level:

In base level reflective technique supports inspection and adaptation. It leads applications to take responsibilities of inspecting middleware and adapting behaviour of its own.

\subsection{Tuple Space Technology}

Tuple Space Middleware is developed by Gelernter in order to overcome frequent disconnections over wireless communications. Tuple Space consists of memory space with globally shared addresses which helps to communicate. Tuple Space technique is similar to template which compares the contents in tuple space during retrieval. Tuple Space is much better in the field where physical mobility and logical mobility involved.

\subsection{Context Aware Technology}

Context Aware Technology is developed to predict the frequent disconnections of communication over mobile devices [10-17] die to the continuous flow of user's mobility. Quality of bandwidth may vary quickly based on user's mobility from one location to another. To predict it middleware displays context info in order to create awareness of dynamic change in application environment $[18,19]$.

\subsection{Event Based Technology}

Event Based Middleware is a process of communication which deals with large environment. It makes use of basic paradigm named Event Notification for communication which includes data (request or message). Events are received in the basis of client interest i.e. client or user have to subscribe a particular event. It also use decoupled paradigm for many to many communication between servers and clients.

\section{Mobile Enterprise}

Mobile Enterprise (as shown in Fig. 5) is an application that helps the user to develop a mobile application in various operating systems. Mobile enterprise environment is straight forward in which user needs a minimal programming knowledge and experience to develop a mobile application. It includes the features like drag and drop to develop an application. 


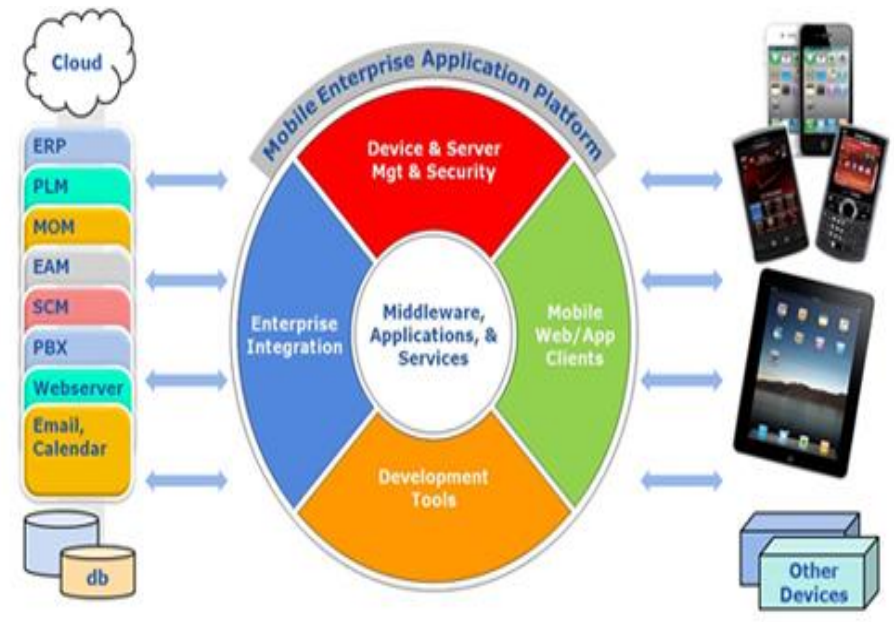

Fig.5. Mobile Enterprise Architecture

$>$ MEAPs allow the user to develop an application without developer background.

$>$ Most of the parts in MEAPs are highly upgradable.

$>$ It allows other devices to consume application from centralized location.

$>$ It cannot be migrated.

In mobility customers MEAPs is an most growing area of interest

\section{Conclusions and Future Work}

This paper explains about several services provided by cloud and the problems faced over mobile cloud application. Mobile Computing Middleware would help to do a better performance over the mobile cloud applications. MCM boosts asynchronous mobile cloud service invocation to perform parallel activities in mobile devices. It also rectifies the scalability issues over the cloud and also it can be magnificent with many web technologies.

Here the one other proposal was upgrading the Mobile Cloud Middleware (MCM) to Mobile Enterprise. Mobile enterprise sets in a cellular network to compete in mobile hosts. This Mobile Enterprise analysis will recreate the Mobile Web Services Mediation Framework (MWSMF) regarding on ESB technology which helps in administrating QoS discovery and some issues over mobile web services. The main motive is to integrate MCM in MWSMF is to cater single interface for mobile web services and mobile cloud services.

\section{Acknowledgements}

The authors are first of all thankful to almighty, and also to the management of VIT University for providing the necessary facilities for presentation of the paper.

\section{References}

[1] Srirama SN, Jarke M, Prinz W. Mobile web services mediation framework. InProceedings of the 2nd workshop on Middleware for service oriented computing: held at the ACM/IFIP/USENIX International Middleware Conference 2007 Nov 26 (pp. 6-11). ACM. 
[2] Google, Inc (2012), "Google Cloud Messaging for Android”, available at: http://developer.android.com/guide/google/gcm/index.html. (accessed January 15, 2017).

[3] HTC Inc. (2000), "Gsm Arena - HTC Desire", available at: http://www.gsmarena.com/htc_desire3077.php. (accessed January 15, 2017).

[4] Paniagua C, Srirama SN, Flores H. Bakabs: managing load of cloud-based web applications from mobiles. InProceedings of the 13th international conference on information integration and web-based applications and services 2011 Dec 5 (pp. 485-490). ACM.

[5] Narasimhan, P. (2010), “Agora: mobile cloud-computing middleware”, available at: http://www.cylab.cmu.edu/research/projects/2010/. (accessed January 15, 2017).

[6] Srirama S, Batrashev O, Vainikko E. Scicloud: scientific computing on the cloud. InProceedings of the 2010 10th IEEE/ACM international conference on cluster, cloud and grid computing 2010 May 17 (pp. 579-580). IEEE Computer Society.

[7] Srirama SN, Flores H, Paniagua C. Zompopo: Mobile calendar prediction based on human activities recognition using the accelerometer and cloud services. InNext Generation Mobile Applications, Services and Technologies (NGMAST), 2011 5th International Conference on 2011 Sep 14 (pp. 63-69). IEEE.

[8] Emmerich W, Aoyama M, Sventek J. The impact of research on middleware technology. ACM SIGOPS Operating Systems Review. 2007 Jan 1;41(1):89-112.

[9] Meier R, Cahill V. Steam: Event-based middleware for wireless ad hoc networks. InDistributed Computing Systems Workshops, 2002. Proceedings. 22nd International Conference on 2002 (pp. 639644). IEEE.

[10] Kumari S, Karuppiah M, Li X, Wu F, Das AK, Odelu V. An enhanced and secure trust-extended authentication mechanism for vehicular ad-hoc networks. Security and Communication Networks. 2016 Nov 25;9(17):4255-71.

[11] Karuppiah M, Kumari S, Li X, Wu F, Das AK, Khan MK, Saravanan R, Basu S. A dynamic id-based generic framework for anonymous authentication scheme for roaming service in global mobility networks. Wireless Personal Communications. 2017 Mar 1;93(2):383-407.

[12] Karuppiah M. Remote user authentication scheme using smart card: a review. International Journal of Internet Protocol Technology. 2016;9(2-3):107-20.

[13] Karuppiah M, Kumari S, Das AK, Li X, Wu F, Basu S. A secure lightweight authentication scheme with user anonymity for roaming service in ubiquitous networks. Security and Communication Networks. 2016 Nov 25;9(17):4192-209.

[14] Li X, Niu J, Karuppiah M, Kumari S, Wu F. Secure and efficient two-factor user authentication scheme with user anonymity for network based e-health care applications. Journal of medical systems. 2016 Dec 1;40(12):268.

[15] Karuppiah M, Saravanan R. A secure authentication scheme with user anonymity for roaming service in global mobility networks. Wireless Personal Communications. 2015 Oct 1;84(3):2055-78.

[16] Karuppiah M, Saravanan R. Cryptanalysis and an improvement of new remote mutual authentication scheme using smart cards. Journal of Discrete Mathematical Sciences and Cryptography. 2015 Sep 3;18(5):623-49.

[17] Wu F, Xu L, Kumari S, Li X, Das AK, Khan MK, Karuppiah M, Baliyan R. A novel and provably secure authentication and key agreement scheme with user anonymity for global mobility networks. Security and Communication Networks. 2016 Nov 10;9(16):3527-42.

[18] Parikh J, Basu A. Effect on System Performance due to Upgradation of 2G/3G System to LTE. International Journal of Wireless and Microwave Technologies (IJWMT). 2016 Jan 8;6(1):11.

[19] Gaur T. A Secure and Efficient Client-Side Encryption Scheme in Cloud Computing. International Journal of Wireless and Microwave Technologies (IJWMT). 2016 Jan 8;6(1):23. 


\section{Authors' Profiles}

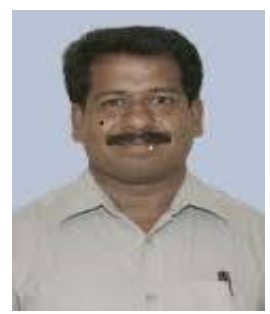

P Thanapal received his B.E. degree in Computer Science and Engineering from Madurai Kamaraj University, Madurai, India, M.E. degree in Computer Science and Engineering from Anna University, Chennai, India and Ph.D. degree in Information Technology and Engineering from VIT University, Vellore, India. He is now an Associate Professor in School of Information Technology and Engineering, VIT University, Vellore, India. His main research interests include cloud computing and web service technologies.

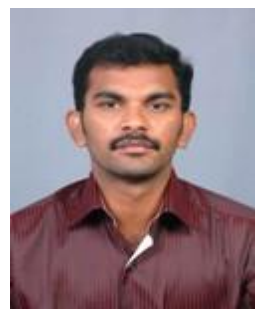

K Marimuthu received his B.E. degree in Computer Science and Engineering from Madurai Kamaraj University, Madurai, India in 2003, M.E. degree in Computer Science and Engineering from Anna University, Chennai, India in 2005, Ph.D. degree in Computer Science and Engineering from VIT University, Vellore, India in 2015. He is now an Associate Professor in School of Computing Science and Engineering, VIT University, Vellore, India. He has published more than 35 research papers in reputed international conferences and journals. He is a life member of Cryptology Research Society of India (CRSI) and Computer Society of India (CSI). His main research interests include cryptography and wireless network security, in particular, authentication and encryption schemes.

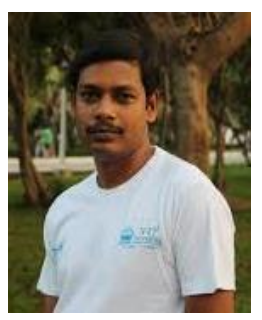

S Rajkumar is currently working as an Assistant professor in school of Computer Science and Engineering, VIT University, Vellore, India. He received his B.E degree in Computer Science and Engineering from Anna University, Chennai, India in 2008, M.E degree in Computer Science and Engineering from Anna University, Chennai, India in 2010. He received $\mathrm{PhD}$ at VIT University, Vellore, India. His research interest includes Digital image processing, Computer Vision, Visual Perception, Object detection, Medical image processing and Infrared image processing. He has published several refereed research papers in various reputed international conferences and journals.

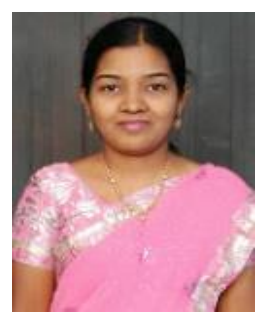

R Niranchana received her B.E degree in Computer Science and Engineering from Anna University, Chennai, India in 2005, M.E degree in Computer Science and Engineering from Anna University, Chennai, India in 2008. She is pursuing PhD at VIT University, Vellore, India. Her main research interests include cryptography and wireless network security, in particular, authentication and encryption schemes.

How to cite this paper: $\mathrm{P}$ Thanapal, $\mathrm{K}$ Marimuthu, S Rajkumar, R Niranchana," Smarter Way to Access Multiple Mobile Cloud Applications without Interoperability Issues", International Journal of Wireless and Microwave Technologies(IJWMT), Vol.7, No.5, pp. 32-39, 2017.DOI: 10.5815/ijwmt.2017.05.04 